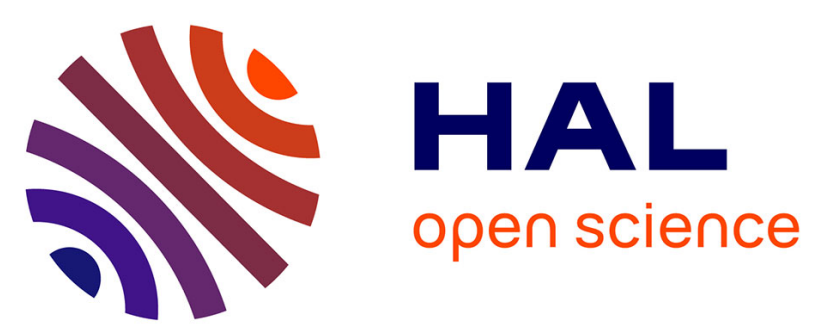

\title{
Binge sucrose-induced neuroadaptations: A focus on the endocannabinoid system
}

\author{
David de Sa Nogueira, Romain Bourdy, Dominique Filliol, Gaëlle Awad, \\ Virginie Andry, Yannick Goumon, Mary C. Olmstead, Katia Befort
}

\section{- To cite this version:}

David de Sa Nogueira, Romain Bourdy, Dominique Filliol, Gaëlle Awad, Virginie Andry, et al.. Binge sucrose-induced neuroadaptations: A focus on the endocannabinoid system. Appetite, 2021, 164, pp.105258. 10.1016/j.appet.2021.105258 . hal-03379174

\section{HAL Id: hal-03379174 \\ https://hal.science/hal-03379174}

Submitted on 14 Oct 2021

HAL is a multi-disciplinary open access archive for the deposit and dissemination of scientific research documents, whether they are published or not. The documents may come from teaching and research institutions in France or abroad, or from public or private research centers.
L'archive ouverte pluridisciplinaire HAL, est destinée au dépôt et à la diffusion de documents scientifiques de niveau recherche, publiés ou non, émanant des établissements d'enseignement et de recherche français ou étrangers, des laboratoires publics ou privés. 


\title{
Binge sucrose-induced neuroadaptations: A focus on the endocannabinoid system
}

\author{
David de Sa Nogueira ${ }^{1,4}$, Romain Bourdy ${ }^{1}$, Dominique Filliol ${ }^{1}$, Gaëlle Awad ${ }^{1}$, Virginie Andry ${ }^{2}$, \\ Yannick Goumon ${ }^{2}$, Mary C. Olmstead ${ }^{3}$, Katia Befort $^{1 *}$
}

\section{Running title: Binge sucrose regulation of the ECS}

${ }^{1}$ Université de Strasbourg, Laboratoire de Neurosciences Cognitives et Adaptatives (LNCA), Centre de la Recherche Nationale Scientifique, 12 rue Goethe, F-67000, Strasbourg France ${ }^{2}$ Institut des Neurosciences Cellulaires et Intégratives (INCI), UPR 3212, CNRS, 8 Allée du Général Rouvillois, 67000, Strasbourg, France

${ }^{3}$ Department of Psychology, Center for Neuroscience Studies, Queen's University, Kingston, Ontario K7L 3N6, Canada

${ }^{4}$ Current address: Brain Health Institute, Rutgers University and Rutgers Biomedical and Health Sciences, Piscataway, New Jersey, USA

\section{*Correspondence}

Katia Befort

Laboratoire de Neurosciences Cognitives et Adaptatives LNCA

Université de Strasbourg Faculté de Psychologie,

12 rue Goethe, F-67000

Strasbourg, France

E-mail: katia.befort@unistra.fr

\begin{abstract}
Abbreviations:
2-AG : 2-arachidonoyglycerol; AEA : anandamide ; BED: Binge-eating disorder; CB1R : cannabinoid receptor 1 ; CB2R : cannabinoid receptor 2 ; CPP: conditioned place preference; dorsal striatum; ECS: endocannabinoid system; HPC: hippocampus; NAc: Nucleus Accumbens ; PFC: prefrontal cortex; RIM : rimonabant [N-(piperidin-1-yl)-5-(4-chlorophenyl)-1-(2,4dichlorophenyl)-4-methyl-1H-pyrazole-3-carboxamide].
\end{abstract}

Key words: Binge eating disorder, sucrose, cannabinoid, gene expression, reward, addiction, conditioned place preference

\section{Words}

Abstract : 277

Introduction : 690

Discussion : 1646 


\begin{abstract}
Binge eating, the defining feature of binge eating disorder (BED), is associated with a number of adverse health outcomes as well as a reduced quality of life. Animals, like humans, selectively binge on highly palatable food suggesting that the behaviour is driven by hedonic, rather than metabolic, signals. Given the links to both reward processing and food intake, this study examined the contribution of the endocannabinoid system (ECS) to binge-like eating in rats. Separate groups were given intermittent (12h) or continuous (24h) access to $10 \%$ sucrose and food over 28 days, with only the $12 \mathrm{~h}$ access group displaying excessive sucrose intake within a discrete period of time (i.e., binge eating). Importantly, this group also exhibited alterations in ECS transcripts and endocannabinoid levels in brain reward regions, including an increase in cannabinoid receptor $1(\mathrm{CB} 1 \mathrm{R}) \mathrm{mRNA}$ in the nucleus accumbens as well as changes in endocannabinoid levels in the prefrontal cortex and hippocampus. We then tested whether different doses (1 and 3mg/kg) of a CB1R antagonist, Rimonabant, modify binge-like intake or the development of a conditioned place preference (CPP) to sucrose. CB1R blockade reduced binge-like intake of sucrose and blocked a sucrose CPP, but only in rats that had undergone 28 days of sucrose consumption. These findings indicate that sucrose bingeing alters the ECS in reward-related areas, modifications that exacerbate the effect of CB1R blockade on sucrose reward. Overall, our results broaden the understanding of neural alterations associated with bingeing eating and demonstrate an important role for CB1R mechanisms in reward processing. In addition, these findings have implications for understanding substance abuse, which is also characterized by excessive and maladaptive intake, pointing towards addictive-like properties of palatable food.
\end{abstract}




\section{Introduction}

Binge-eating disorder (BED), now listed as an independent disorder in DSM-5, is characterized by uncontrollable episodes of eating within a discrete period of time, not followed by compensatory behaviors such as purging or physical exercise (APA, 2013). BED is the most common of all eating disorders, affecting approximately $3 \%$ of the general population (Hudson et al., 2007; Kessler et al., 2013; Solmi et al., 2016). Binge eating also occurs in other eating disorders (Hudson et al., 2007) and is a likely contributor to the growing obesity epidemic (Stojek \& MacKillop, 2017). Although binge eating is linked to serious medical complications, including increased risk for other psychiatric disorders, treatment options for BED are minimal (Hutson et al., 2018). Advancement in this area depends on understanding the etiology and mechanisms that drive binge eating.

Regulation of food intake in both humans and animals is closely linked to activity in the endocannabinoid system (ECS) (Ceccarini et al., 2016), the endogenous system activated by $\Delta$ 9-tetrahydrocannabinol in the Cannabis Sativa plant. For example, blockade of the cannabinoid receptor 1 (CB1R) reduces food intake (Arnone et al., 1997; Simiand et al., 1998; Colombo et al., 2002), specifically, binge-like eating in rats (Parylak et al., 2012; Scherma et al., 2013; Dore et al., 2014), and decreases scores on a binge eating scale in humans (Pataky et al., 2013). Most telling, infusions of the endogenous cannabinoid, 2-arachidonoyglycerol (2-AG), directly into the nucleus accumbens (NAc), dose-dependently increases feeding in rats, an effect that is blocked by CB1R antagonism (Kirkham et al., 2002). Results from these animal studies linking the ECS to excessive eating is not surprising given that cannabis is well known to promote eating in humans (Abel, 1975; Williams et al., 1998; Koch et al., 2015). 
In addition to the direct effects of cannabinoid compounds on feeding, maladaptive eating patterns, themselves, may disrupt ECS function (Gaetani et al., 2008). The specific details are difficult to disentangle in that a number of studies have yielded conflicting results. In humans, BED is associated with increased plasma levels of the endogenous cannabinoid anandamide (AEA), but not 2-AG, although the same pattern was observed in anorexia nervosa (Monteleone et al., 2005). In animal models, bingeing on a high fat diet decreases AEA levels in the dorsal striatum (DS), amygdala, and hippocampus (HPC), but increases 2-AG levels in the HPC (Satta et al., 2018). Food restriction (Dazzi et al., 2014) and continuous access to a high-fat diet (Blanco-Gandía, Aracil-Fernández, et al., 2017) decrease both gene and protein expression of CB1Rs in the prefrontal cortex (PFC), whereas excessive intake of fat/sweet pellets increases CB1R expression in the same region (Mancino et al., 2015). In the NAc, CB1Rs are upregulated, but CB1R transcripts are decreased, following either binge or continuous intake of high fat foods (Blanco-Gandía, Aracil-Fernández, et al., 2017; Blanco-Gandía, Cantacorps, et al., 2017). These seemingly contradictory findings likely reflect the use of different feeding protocols and/or type of palatable food, particularly as the biological mechanisms underlying fat and sucrose bingeing are distinct (Wong et al., 2009).

The primary goal of this study was to investigate, in more detail, the relationship between ECS function and binge eating. We focused on sweet foods, using a validated model of sucrose bingeing in rats (Avena et al., 2005; Avena, 2007; Avena, Bocarsly, et al., 2008; Avena, Rada, et al., 2008), in order to isolate the effects of this commodity from other palatable foods. A link between binge eating and the ECS likely occurs through brain reward circuits (Maldonado et al., 2006) in that binge eating is driven by hedonic, rather than metabolic, signals. Thus, our first set of experiments examined whether binge eating alters gene expression of CB1R and cannabinoid 
type 2 receptor (CB2R), as well as endocannabinoid levels (2-AG and AEA), in brain reward sites including the NAc, DS, PFC, and HPC. We also measured enzyme transcripts for endocannabinoid synthesis (NAPE-PLD/DAGL $\alpha$ ) and degradation (FAAH/ MAGL) in all four regions. Based on our observation that sucrose bingeing alters CB1R gene expression, we extended these studies by testing whether blockade of CB1Rs alters sucrose bingeing or the rewarding effect of sucrose in the conditioned place preference (CPP) paradigm.

\section{Materials and methods}

\subsection{Subjects}

Two hundred and twenty-three male Wistar rats (Charles River Laboratories, Montréal QC and Janvier Laboratories, France), weighing $175 \mathrm{~g}$ at arrival, were habituated for two weeks to housing conditions in a temperature and humidity-controlled environment on a reverse $12 \mathrm{~h}$ light/dark cycle (lights OFF at 4:00 AM). Rats were group housed in standard polycarbonate cages with ad libitum access to food and water until 1 day before behavioral experiments. All animals were then single housed to provide accurate measures of individual food and solution consumption. All procedures and animal care were performed according to the European Union laws for animal studies and were in accordance with the guidelines for the ethical use of animals, outlined by the Canadian Council on Animal Care. Experiments were approved by the institutional ethics committee CREMEAS (Comité d'Éthique pour l'Expérimentation Animale de Strasboug, France) (APAFIS\#2019070816359145) and the Queen's University Animal Care Committee. A timeline displaying the sequence of experimental procedures for each set of experiments is shown in Figure 1.

\subsection{Apparatus}


Body weight and food consumption (g) were recorded with a standard scale. Liquid solutions were presented to rats in $100 \mathrm{ml}$ graduated glass drinking bottles fitted with rubber stoppers containing ball-tipped sipper tubes (Ancare Inc., Montreal, QUE) or in bottles (Techniplast, Milan, Italy) that were weighed to collect consumption measures. Sucrose was mixed in tap water, measured as $\mathrm{g} / \mathrm{L}$. Solution concentration was $10 \%$ sucrose. The $\mathrm{CB}_{1}$ receptor antagonist, rimonabant [N-(piperidin-1-yl)-5-(4-chlorophenyl)-1-(2,4-dichlorophenyl)-4-methyl1H-pyrazole-3-carboxamide] (RIM) (Cayman Chemicals, Burlington, ON) was dissolved in one drop vehicle of $0.3 \%$ polysorbate Tween-80 (Sigma-Aldrich Chemicals, St. Louis, MO) dissolved in $0.9 \%$ saline. Fresh solutions of $1 \mathrm{mg} / \mathrm{ml}$ and $3 \mathrm{mg} / \mathrm{ml}$ were prepared each day.

The CPP apparatus was made of plexiglass and consisted of two large compartments (46 x 46 x $30 \mathrm{~cm})$ connected by a tunnel $(19 \times 38 \times 30 \mathrm{~cm})$. The two large compartments differed in wall colour (black and white stripes or solid white) and floor texture (striated or bumpy). The tunnel walls were clear plexiglass and the floor was made of sheet metal, spray painted with a matt white finish. Guillotine doors, that could be raised or lowered, separated the tunnel from each compartment. Cameras were mounted directly above each set of boxes so that the rats' movements throughout the entire compartment could be monitored and recorded. Movement was tracked using the video tracking software EthoVisonXT (Noldus Information Technology b.v. Wageningen, The Netherlands), which detects and extracts the size and position of the subjects from the digital image captured by the camera.

\subsection{Procedures}

\subsubsection{Effects of sucrose bingeing on neuroadaptations in the ECS}

\subsubsection{Sucrose consumption}


Eighty-three rats were assigned to one of three groups that determined daily access to sweet solution and food over 28 days (Figure 1, experiment 1).

For the primary experimental group, $12 \mathrm{~h}$ sucrose $(\mathrm{n}=28)$, chow was removed from the cages $12 \mathrm{~h}$ prior to the first access day. Then, beginning $4 \mathrm{~h}$ after the onset of the active cycle, rats were provided with $12 \mathrm{~h}$ access to standard chow and sucrose solution. The first control group (12h food, $\mathrm{n}=28$ ) underwent the same protocol with no sucrose, providing a control for food restriction. The second control group (24h sucrose; $n=27$ ) had continuous access to standard chow and sucrose solution, which was introduced on day 1 (4h after the onset of the active cycle), providing a control for sucrose exposure.

All animals were weighed daily, prior to the presentation of solution and/or food. For intermittent access groups (12h sucrose, $12 \mathrm{~h}$ food), solution intake (ml) was measured $1 \mathrm{~h}$ after presentation and on removal (12h). For animals in the continuous access group (24h sucrose), solution intake was measured 1,12 , and $24 \mathrm{~h}$ after presentation. Food intake $(\mathrm{g})$ was measured at the end of the access period (12h or $24 \mathrm{~h})$.

\subsubsection{Brain dissection}

The day following the final sucrose access session, rats were given an overdose of pentobarbital (182 mg/kg, i.p), as required by regulations of the local animal ethics committee, followed by decapitation to perform brain extraction. This time point was selected to minimize acute effects of the last sucrose exposure and to avoid potential circadian effects. Coronal brain slices, $1 \mathrm{~mm}$ thick, were cut using a brain matrix chilled on ice (Harvard apparatus, Holliston, MA, USA). Structures of interest were collected according to the rat brain stereotaxic atlas (Paxinos \& Watson, 2007) by dissection (dorsal HPC, -2 to 4mm from bregma, 2 slices) or using punches (PFC, $3 \mathrm{~mm}$ puncher, 4.68 to $2.5 \mathrm{~mm}$ from bregma, 2 slices; NAc, $1.9 \mathrm{~mm}$ puncher, 3 to 
0.6mm from bregma, bilateral, 2 slices; DS, 3mm puncher, 2 to $-0.96 \mathrm{~mm}$ from bregma, bilateral, 3 slices). Samples were immediately frozen on dry ice and kept at $-80^{\circ} \mathrm{C}$. All extractions were performed in less than 20 min to avoid increased levels of AEA (Schmid et al., 1995).

\subsubsection{Quantitative real-time PCR}

Total RNA was extracted using Ribozol (VWR, Fontenay-sous-bois, France) according to the manufacturer's instructions. RNA quality and quantity were measured with a NanoVue $\mathrm{TM}^{\mathrm{TM}}$ (GE healthcare) spectrophotometer (GE healthcare). Reverse transcription was performed on 750 ng of total RNA with iScript (iScript ${ }^{\mathrm{TM}} \mathrm{cDNA}$ Synthesis Kit, Biorad, France). Real-time PCR was performed in triplicate using a CFX96 Touch $^{\mathrm{TM}}$ apparatus (Biorad, France) and Sso Advanced $^{\mathrm{TM}}$ Universal SYBR Green supermix (Biorad, France). Thermal cycling parameters were $30 \mathrm{sec}$ at $95^{\circ} \mathrm{C}$ followed by 40 amplification cycles of $5 \mathrm{sec}$ at $95^{\circ} \mathrm{C}$ and $45 \mathrm{sec}$ at $60^{\circ} \mathrm{C}$. Primer sequences for all tested genes are provided in Table 1. Expression levels were normalized to $R p l p O$ housekeeping gene levels, classically used for brain or food related studies (Silberberg et al., 2009; Li et al., 2014) and compared between controls and treated samples using the $2^{-\Delta \Delta C t}$ method (Livak, 2001).

\subsubsection{Mass spectrometry}

PFC, HPC, NAc and DS tissues (n=7-8/group) were sonicated in $200 \mu 1$ of $\mathrm{H}_{2} \mathrm{O}$ with a Vibra Cell apparatus (2 times 5 s, 90W; Sonics, Newtown, U.S.A.) and the homogenate was centrifuged $\left(20,000 \mathrm{~g}, 30 \mathrm{~min}, 4^{\circ} \mathrm{C}\right)$ to recover the supernatant. Protein concentration was determined (Protein Assay, Bio-Rad, Marnes-la-Coquette, France) and $150 \mu \mathrm{l}$ of the supernatant was mixed with $50 \mu \mathrm{l}$ of acetonitrile (ACN) $100 \%$ containing 400.26 pmol of D8-2-AG (sc480539; Santa Cruz, Heidelberg, Germany) and 100.15 pmol of D4-AEA (Tocris/Biotechne, Lille, France). Samples were centrifuged $\left(20,000 \mathrm{~g}\right.$ for $\left.30 \mathrm{~min}, 4^{\circ} \mathrm{C}\right)$ and the supernatants 
collected and evaporated to dryness. Samples were re-suspended in $20 \mu \mathrm{l}$ of ACN 30\% / $\mathrm{H}_{2} \mathrm{O}$ $69.9 \%$ / formic acid $0.1 \%(\mathrm{v} / \mathrm{v} / \mathrm{v})$. Analyses were performed on a Dionex Ultimate 3000 HPLC system coupled with a triple quadrupole Endura mass spectrometer (Thermo Electron, San Jose, USA). The system was controlled by Xcalibur v. 2.0 software. Samples $(3 \mu \mathrm{l})$ were loaded onto a microbore C18 ODS column (1x100 mm, $3 \mu$ m UniJet microbore ODS, ref MF8949, BioAnalytical Systems Inc., West Lafayette, U.S.A.) heated at $40^{\circ} \mathrm{C}$. The presence of 2-AG, AEA, D8-2-AG and D5-AEA was studied using the multiple reaction monitoring mode (MRM). Elution was performed at a flow rate of $50 \mu 1 / \mathrm{min}$ by applying a linear gradient of mobile phases A/B. Mobile phase A corresponded to ACN 1\% / $\mathrm{H}_{2} \mathrm{O} 98.9 \%$ / formic acid $0.1 \%(\mathrm{v} / \mathrm{v} / \mathrm{v}$ ), whereas mobile phase B was ACN 99.9\% / formic acid 0.1\% (v/v) (see details in Supplemental Table 1).

Electrospray ionization was achieved in the positive mode (spray voltage set at 3,500 V). Nitrogen was used as the nebulizer gas. Desolvation (nitrogen) sheath gas was set to 10 Arb and Aux gas was set to $5 \mathrm{Arb}$. The Ion transfer tube was heated at $287^{\circ} \mathrm{C}$. Q1 and Q2 resolutions were set at $0.7 \mathrm{FWHM}$, whereas collision gas (CID, argon) was set to 2 mTorr. Identification of the compounds was based on precursor ion, selective fragment ions and retention times obtained for 2-AG, AEA, D8-2-AG and D5-AEA. Selection of the monitored transitions and optimization of collision energy and RF Lens parameters were manually determined (see Supplemental Table 2). Qualification and quantification were performed with the MRM mode and quantification was obtained using Quan Browser software (Thermo Scientific). All amounts of endocannabinoids measured in samples fit within the standard curve limits, with typical analytical ranges from $1 \mathrm{fmol}-100 \mathrm{pmol}$ to $150 \mathrm{fmol}-100$ pmol. Precision (CV\% between repeated injections of the same sample) values were $<1 \%$ for same-day measurements and $<5 \%$ 
for inter-day measurements. The amount of 2-AG (nmol) and AEA (pmol) were normalized according to protein levels (mg).

\subsubsection{Effects of CB1 antagonism on sucrose bingeing}

Sixty rats were randomly assigned to $12 \mathrm{~h}$ or $24 \mathrm{~h}$ sucrose groups, which determined access to food and sucrose solution over 28 days using the protocol described previously (2.3.1.2). Within each access group, rats were randomly assigned to a drug dose $(0,1$, or $3 \mathrm{mg} / \mathrm{kg}$ RIM) ( $n=10$ each), and were then injected intraperitoneally (i.p.) with drug 30 min before the start of each sucrose access sessions on days 1-5 and 24-28 (Figure 1, experiment 2). No injections were administered on days 6-23 inclusive. Doses and timing of pre-treatment with RIM were based on previous studies showing behavioural effects of the drug, including an impact on food intake (Blasio, et al., 2014) and binge eating (Scherma, et al., 2013), but also on social play (Achterberg, et al., 2016), cocaine responses (Chaperon, et al., 1998; De Vries, et al., 2001; Yu, et al., 2011), and morphine reward (De Carvalho, et al., 2014).

\subsubsection{Effects of CB1 antagonism on sucrose reward}

Twenty-four rats, divided randomly into three drug doses ( $n=8$ each) were used to examine the effect of RIM on sucrose reward in the CPP paradigm (Figure 1, experiment 3). Rats were food restricted for $48 \mathrm{~h}$ prior to and throughout the CPP protocol. On day 1 (habituation), rats were placed in the tunnel and had free access to all three compartments for 30 min. Over the next 10 days, rats were confined to one test compartment or the other for $30 \mathrm{~min}$ where they had access to a water bottle containing either tap water or $20 \%$ sucrose solution. The assignment of sucrose-paired compartment and order of conditioning sessions (water- versus sucrose-paired) were counterbalanced within groups. Rats were injected with RIM (0, 1 , or 3 $\mathrm{mg} / \mathrm{kg}$ i.p.) $30 \mathrm{~min}$ prior to conditioning in the sucrose-paired compartment and with vehicle 
solution (1 ml/kg i.p.) $30 \mathrm{~min}$ prior to conditioning in the water-paired compartment. The amount of fluid consumed during each conditioning session was measured. On test day, rats were placed in the tunnel and allowed free access to the entire apparatus. Water bottles were removed from both compartments and the time spent in each compartment was recorded across the 30 -min session.

In a separate experiment, forty-eight rats, randomly divided into two access groups (12h and $24 \mathrm{~h}$ sucrose), underwent sucrose consumption sessions using the protocol described in 2.3.1.2 (Figure 1, experiment 4). Rats were then randomly divided into three drug groups $(n=8$ each) and tested for a sucrose CPP as outlined above (0, 1, and $3 \mathrm{mg} / \mathrm{kg} \mathrm{RIM})$.

\section{$2.4 \quad$ Statistical analysis}

Solution and food intake (measured per body weight as $\mathrm{ml} / \mathrm{g}$ and $\mathrm{g} / \mathrm{g}$ ) and weight were analyzed using a repeated-measures analysis of variance (ANOVA) in the mixed effect way (Afex package from R, Rstudio v1.3.1093). For the fixed effects part of the model, the explanatory variables were session as the repeated measure and sucrose access group and drug as betweensubjects measures. The random effect part of the model accounts for the individual variation of the subjects across the sessions. In cases in which sphericity was violated (Machley's $p<0.001$ ), the Greenhouse-Geisser correction was used. Post hoc analysis were conducted by multiple pairwise comparisons using the Sidak method for correction (lsmeans package from R).

Biochemical data were analyzed using ANOVA to assess group differences in ECS neuroadaptations, with separate analyses conducted in each brain region. Significant interactions were followed up with simple main-effects analyses and multiple pairwise comparisons using a Bonferroni correction (GraphPad Prism software). 
For CPP data, consumption of solution (sucrose and water) during conditioning and time spent in each compartment during testing were analyzed using planned orthogonal comparisons, as described previously (SPSS v27) (Smail-Crevier et al., 2018; Grenier et al., 2019). A primary advantage of this technique is that it minimizes the number of comparisons to those of interest; because each comparison is independent and tests a unique hypothesis, it can be carried out regardless of the outcome of the overall ANOVA and no correction is made for multiple tests. As such a 5\% risk of type I error is accepted for each comparison. Levene's F test was used to examine the assumption of homogeneity of variance and analyses were adjusted if significant. Significance level for all tests was set at $p \leq .05$.

\section{Results}

\subsection{Effects of sucrose bingeing on neuroadaptations in the ECS}

\subsubsection{Sucrose consumption}

Figure 2 shows data from the sucrose consumption sessions, with intake during the $1^{\text {st }}$ hour of access being significantly higher in rats given intermittent, rather than continuous, access to sucrose, $F(1,32)=55.11, p<.001$, general $\eta 2=.39$ (group) (Figure 2A). The main effect of session, $F(9.84,314.85)=4.41, p<.001$, general $\eta 2=.08$, as well as the session $\mathrm{x}$ group interaction, $F(9.84,314.85)=4.95, p<.001$, general $\eta 2=.09$, were also statistically significant. With the exception of session 1 , intake was higher in the $12 \mathrm{~h}$, compared to the $24 \mathrm{~h}$, sucrose group (post-hoc ps <.0001), verifying binge-like intake in rats given intermittent access to sucrose. In contrast, there was no significant difference in daily sucrose intake for rats in the $12 \mathrm{~h}$ and $24 \mathrm{~h}$ sucrose groups, $F(1,33)=0.46, p=.501$, general $\eta 2=.01$ (Figure 2B). Daily solution intake varied across sessions, $F(5.44,179.55)=3.04, p<.01$, general $\eta 2=.04$, but the effect did not interact with group, $F(5.44,179.55))=1.70, p=.13$, general $\eta 2=.02$. 
Figure 2C shows that food intake of all groups declined across sessions, $F(11.92,453.05)$ $=132.49, p<.001$, general $\eta 2=.71$, with higher overall intake in the food only group, $F(2,38)=$ $70.77, p<.001$, partial $\eta 2=.53$. The significant session $\mathrm{x}$ group interaction, $F(23.84,453.05)=$ 6.97, $p<.001$, partial $\eta 2=.20$, reflected higher food intake in the $12 \mathrm{~h}$ food group beginning on day 2 compared to both sucrose access groups, with no significant difference between the other two groups across sessions $(p s>.05)$. Finally, body weight increased across sessions in all groups, $F(2.27,147.36)=2176.71, p<.001$, general $\eta 2=.77$. The significant main effect of group, $F(2,65)$ $=8.18, p<.001$, general $\eta 2=.18$, and session $\mathrm{x}$ group interaction, $F(4.53,147.36)=4.55, p=.001$, general $\eta 2=.014$, were due to higher body weight in the 24 h sucrose group beginning on day 2 , compared to both $12 \mathrm{~h}$ sucrose and $12 \mathrm{~h}$ food groups $(p s<.05)$, and no difference in body weight of $12 \mathrm{~h}$ sucrose and $12 \mathrm{~h}$ food groups across sessions ( $p \mathrm{~s}>.05)$ (Figure 2D).

\subsubsection{Gene expression}

Figure 3 shows the effects of sucrose bingeing on gene expression in the ECS throughout brain reward sites. Cnrl (CB1R coding gene) expression was increased in the NAc of rats in the 12h sucrose group, compared to either $12 \mathrm{~h}$ food or $24 \mathrm{~h}$ sucrose groups, $F(2,24)=4.90, p<.05$

(Figure 3A). There were no differences in $C n r l$ expression in other regions (PFC: $F(2,20)=.84$, $p=.45 ; \mathrm{DS}: F(2,21)=.041, p=.96 ; \mathrm{HPC}: F(2,20)=1.36, p=.28)$ and no differences in $C n r 2$ expression across any site $(\mathrm{PFC}: F(2,20)=.77, p=.47 ; \mathrm{NAc:} F(2,15)=.44, p=.65 ; \mathrm{DS}: F(2,19)$ $=1.27, p=.30 ;$ HPC: $F(2,25)=1.26, p=.30)$.

Further analysis showed Faah expression was decreased in the NAc, only in the $24 \mathrm{~h}$ sucrose group, $F(2,21)=4.03, p<.05$ (Figure 3B), whereas Dagla expression in this region was decreased in both sucrose groups, $F(2,21)=10.22, p<.001$ (Figure 3C). There were no 
significant differences in enzyme expression in the PFC $($ Faah: $F(2,20)=.09, p=.91$; Dagla: $F(2,20)=.58, p=.57), \mathrm{DS}(F a a h: F(2,18)=.57, p=.58 ;$ Dagl $: F(2,18)=.13, p=.88)$, or HPC $($ Faah: $F(2,20)=.87, p=.43 ;$ Dagl $: F(2,20)=1.67, p=.21)$. Pearson's correlation analysis revealed no significant relationship between sucrose intake during the first hour of access and regulated genes for any group (data not shown).

\subsubsection{Endocannabinoid levels}

In assessing endocannabinoid levels following sucrose bingeing, we observed an increase of AEA in the PFC of the $12 \mathrm{~h}$ sucrose group, $F(2,21)=4.05, p<.05)$, with no other differences in this measure $(\mathrm{NAc:} F(2,21)=.04, p=.96$; DS: $F(2,21)=.16, p=.85 ; \mathrm{HPC}: F(2,18)=.13, p=$ .87) (Figure 4A). In contrast, $2-\mathrm{AG}$ was decreased in the HPC of the $12 \mathrm{~h}$ sucrose group, $F(2,18)$ $=3.75, p<.05)$, with no group differences in other brain regions $(\mathrm{PFC}: F(2,21)=.49, p=.62$; NAc: $F(2,21)=.20, p=.82$; DS: $F(2,21)=.21, p=.81)$ (Figure 4B). Pearson's correlation analysis revealed no significant relationship between sucrose intake during the first hour of access and regulated eCB levels for any group (data not shown). We did not perform correlation analyses between gene expression and $\mathrm{eCB}$ levels as these measures were obtained on two distinct cohorts.

\subsection{Effects of CB1 antagonism on sucrose consumption}

Figure 5A (left panel) shows that sucrose intake during the first hour of access increased across days, with the $12 \mathrm{~h}$ sucrose groups consuming significantly more solution than the $24 \mathrm{~h}$ sucrose groups. These effects were verified, statistically, by a significant session $\mathrm{x}$ group interaction, $F(12.74,688.12)=10.22, p<.001$, general $\eta 2=.11$, as well as significant main effects of session, $F(12.74,688.12)=20.70, p<.001$, general $\eta 2=.19$, and group, $F(1,54)=$ 
268.33, $p<.001$, general $\eta 2=.65$. The main effect of drug, $F(2,54)=3.40, p<.05$, general $\eta 2$ $=.04$, reflected decreased intake in sucrose groups following RIM, compared to vehicle, administration $(p s<.01)$. The session $x$ group $x$ drug interaction was statistically significant, $F(25.49,688.12)=1.88, p<.01$, general $\eta 2=.042$. Post-hoc test revealed that intake of the $12 \mathrm{~h}$ sucrose vehicle group was significantly higher than the 24 h sucrose vehicle group $(p<.0001)$; in the $12 \mathrm{~h}$ sucrose groups, intake was significantly lower following RIM 3, compared to RIM 1, treatment $(p=0.0002)$ and there was no significant difference between intake of the $24 \mathrm{~h}$ sucrose RIM 1 and 24h sucrose RIM 3 groups. The effects of RIM on binge intake were examined, in more detail, by comparing total intake during the first hour of days $1-5$ and days $24-28$ in the $12 \mathrm{~h}$ sucrose group (Figure 5A, right panel). This analysis revealed no significant differences across the first 5 sessions, $F(2,96.8)=1.23, p=.29$, but a significant effect of drug during the final 5 sessions, $F(295.2)=8.56, p<.001$. Both doses of RIM significantly decreased sucrose intake during the first hour of these 5 sessions, compared to vehicle injections (post-hoc $p s<.01$ ).

Daily sucrose intake also increased across sessions, $F(8.32,449.33)=81.80, p<.001$, general $\eta 2=.42$ (Figure 5B, top panel) although there were no group or drug differences on this measure and no interaction between the two (main effects and interaction, $p \mathrm{~s}>.05$ ). The session $\mathrm{x}$ group $\mathrm{x}$ drug interaction was significant $(F(16.64,449.33)=1.88, p=.02$, general $\eta 2=.03)$, as well as the session $\mathrm{x}$ group interaction $(F(8.32,449.33)=2.94, p<.01$, general $\eta 2=.03)$, suggesting that a change in sucrose intake across days was dependent on both access condition and drug dose. Post-hoc analyses revealed lower intake in the $12 \mathrm{~h}$, compared to the $24 \mathrm{~h}$, sucrose group following vehicle administration $(p<.0001)$, but no significant differences between these groups following RIM administration. In addition, both doses of RIM increased intake, compared to vehicle, in the $12 \mathrm{~h}$ sucrose group ( $p s<.0001$ ), with no significant differences 
between intake of the two RIM-treated groups. The higher (RIM 3), but not the lower (RIM 1) drug dose decreased intake in the $24 \mathrm{~h}$ sucrose group, compared to vehicle -treated rats $(p<$ .0001 ), and intake of the $24 \mathrm{~h}$ sucrose group administered RIM 3 was significantly lower than intake of the $24 \mathrm{~h}$ sucrose group receiving RIM $1(p=.0006)$.

Figure 5B (middle panel) shows that food intake declined across sessions in all groups, $F(13.21,713.24)=38.50, p<.001$, general $\eta 2=.22$, with significant interactions between session and group, $F(13.21,713.24)=2.14, p=.01$, general $\eta 2=.02$; and session and drug dose $F(26.42,713.24)=1.56, p=.04$, general $\eta 2=.02$. The main effect of group, $F(1,54)=10.61, p=$ .002 , general $\eta 2=.11$, was due to higher food intake in the $12 \mathrm{~h}$ sucrose groups $(p<.0001)$. Post-hoc tests revealed that the highest dose of RIM decreased food intake, compared to vehicle injections, in the $12 \mathrm{~h}$ sucrose groups $(p s<.0001)$. In contrast, RIM 1 increased food intake in the 24h sucrose groups $(p=0.02)$.

As shown in Figure 5B (bottom panel), body weight increased across sessions in all groups, $F(3.36,186.85)=559.92, p<.001$, general $\eta 2=.7$, and the effect interacted with drug, $F(6.92,186.85)=3.20, p=.003$, general $\eta 2=.03$, indicating that the impact of drug on body weight varied across sessions. Post-hoc tests revealed lower body weights in the $12 \mathrm{~h}$ sucrose group administered RIM 3, compared to RIM 1, on session average $(p<.0001)$. In the $24 \mathrm{~h}$ sucrose groups, vehicle-treated rats were heavier than rats treated with the higher and lower RIM doses $(p s<.0001)$. None of the other main effects or interactions were statistically significant.

\subsection{Effects of CB1 antagonism on sucrose reward}

Figure 6A shows that all rats consumed significantly more sucrose than water across the CPP conditioning sessions (vehicle, $t(42)=8.44, \mathrm{RIM} 1, t(42)=7.71, \mathrm{RIM} 3, t(42)=4.90$, all $p \mathrm{~s}$ $<.01)$. The significant main effect drug, $F(2,42)=3.57, p<.05$, partial $\eta 2=.15$, indicated that 
RIM significantly reduced overall consumption during conditioning sessions, with RIM 3 reducing total sucrose intake, compared to the vehicle group (post-hoc, $p<.05$ ). In contrast, RIM had no effect on the development of a sucrose CPP, $F(2,42)=.93, p=.40$, partial $\eta 2=.15$, in that all three groups showed a preference for the sucrose-paired compartment $($ vehicle, $t(42)=$

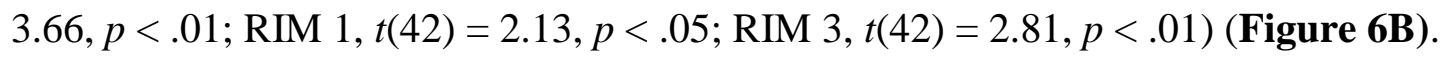

We also tested the effects of RIM on a sucrose CPP in rats given either intermittent or continuous access to sucrose over 28 days. As with the previous two cohorts (see 3.1.1 and 3.2.), the $12 \mathrm{~h}$ sucrose group developed binge-like intake (i.e., increased intake during the first hour of access) that was absent in the $24 \mathrm{~h}$ group (data not shown). During subsequent CPP conditioning sessions, both groups consumed more sucrose than water across the 5 days (12h sucrose: vehicle, $t(84)=6.62, \mathrm{RIM} 1, t(84)=5.28, \operatorname{RIM} 3, t(84)=6.33 ; 12 \mathrm{~h}$ sucrose: vehicle, $t(84)=7.22, \mathrm{RIM} 1$, $t(84)=5.48$, RIM 3, $t(84)=5.01$, all $p s<.01)$, with no group $F(1,84)=.19, p=.66$, partial $\eta 2=$ .01 or drug, $F(2,84)=1.38, p=.26$, partial $\eta 2=.03$, differences on this measure (Figure 6C). During CPP testing (Figure 6D), however, only two groups displayed a significant preference for the sucrose-paired compartment: $24 \mathrm{~h}$ sucrose plus vehicle, $t(84)=4.11, p<.01$, and $24 \mathrm{~h}$ sucrose plus RIM $1, t(84)=1.99, p<.05$. None of the other rats showed a preference for either the sucrose- or water-paired side during testing, all $p \mathrm{~s}>.05$.

\section{Discussion}

Our study confirms an important role for the ECS, specifically CB1Rs, in sucrose bingeing. More specifically, binge-like intake of sucrose modified ECS signaling in brain reward regions and $\mathrm{CB} 1 \mathrm{R}$ blockade reduced this behavior in rats. $\mathrm{CB} 1 \mathrm{R}$ antagonism, on its own, had no effect on the development of a sucrose CPP but blocked this effect in animals that had already consumed excessive amounts of sucrose. We propose that excessive sucrose intake modifies 
ECS signals related to reward processing, rendering animals vulnerable to CB1R antagonism in the CPP paradigm. Taken together, our findings further the understanding of mechanisms sustaining sucrose bingeing, including how these relate to reward processing in brain regions that are also implicated in drug addiction.

Our study provides the first evidence that CB1R antagonism reduces binge-like intake of a sucrose solution, measured in animal models as excessive intake during the first hour of access (Avena et al., 2005; Smail-Crevier et al., 2018; Maracle et al., 2019). The effect was manifested, specifically, during the final sessions of intermittent sucrose access following administration of RIM. Our findings corroborates previous evidence that CB1R blockade in rats dose-dependently decreases bingeing of palatable foods that are high in sugar (Dore et al., 2014), sugar and fat (Parylak et al., 2012), or fat only (Scherma et al., 2013). The reduction in binge-like eating following RIM administration may reflect a general decline in palatable food intake (Dore et al., 2014), although we did not observe an effect of drug on daily intake of sucrose, or on hourly intake in rats that did not exhibit binge-like eating (i.e., 24h sucrose group). Admittedly, these measures may be less sensitive to disruption by CB1R antagonism in that binge-like eating is an exaggerated response that occurs within a short period of time and, given the half-life of the drug, effects would have dissipated by two or three hours into the sessions (Jarbe, et al., 2010). Thus, although some details require further clarification, it is becoming increasingly clear that CB1Rs play an important role in over consumption of sweet foods.

Our post-mortem assessment revealed an interesting pattern of ECS-related changes following excessive sucrose intake. This included an upregulation of CB1R gene expression in the NAc, increased AEA levels in the PFC, and decreased 2-AG levels in the HPC; importantly, these changes were only apparent in rats that exhibited binge-like intake of sucrose. In contrast to 
Binge sucrose regulation of the ECS

our findings, binge intake of high fat or high fat-high sugar foods decreased CB1R gene expression in the NAc (Bello et al., 2012; Blanco-Gandía, Cantacorps, et al., 2017), providing further evidence that the biological consequences of high fat and high sugar diets are dissociable (Avena, 2010). Our study is the first to assess brain endocannabinoid levels in an animal model of sucrose bingeing, supporting clinical reports of alterations in blood AEA levels in BED patients (Monteleone et al., 2005). We also observed sucrose-induced decreases in ECS enzymes (FAAH and MAGL) that were not restricted to bingeing rats, suggesting that excessive sucrose intake, itself, may impact ECS function. Not surprisingly, these changes were limited to the NAc, an area that is closely associated with consumption of palatable food (Kelley et al., 2005). A role for the ECS in sucrose bingeing likely occurs through an interaction with brain reward systems. Indeed, both palatable food (Joseph \& Hodges, 1990; Phillips et al., 1993) and endocannabinoids (Solinas et al., 2006) increase dopamine (DA) release in the NAc shell, with the latter acting via CB1Rs in this region (Sperlágh et al., 2009). Moreover, NAc neurons encode signals related to food reward and this effect is attenuated by CB1R antagonism (Hernandez \& Cheer, 2012; Thoeni et al., 2020). CB1Rs appear to form heteromers with NAc opioid receptors (Rodriguez et al., 2001; Rios et al., 2006), which mediate the hedonic aspects of sweet foods (Peciña \& Berridge, 2005). Intriguingly, excessive sucrose intake also increases binding of both mu opioid and DA D1 receptors in the NAc (Colantuoni et al., 2001), pointing to an interaction between multiple transmitter systems in the control of palatable food intake. Finally, an ECS contribution to food reward may reflect a more general role for this system in reward processing in that both CB1Rs and endocannabinoids in the NAc play a role in reward linked to social interaction (Trezza \& Vanderschuren, 2008; Trezza et al., 2012; Wei et al., 2015; Manduca et al., 2016) and to a morphine CPP (Khaleghzadeh-Ahangar \& Haghparast, 2015). 
Modifications in ECS processes following extended sucrose access may explain our seemingly contradictory findings in the CPP paradigm. More specifically, in animals with no prior exposure to sucrose (i.e., rats that had no gone through the sucrose binge experiment), CB1R antagonism did not alter the development of a sucrose CPP. In contrast, the highest dose of RIM eliminated a CPP in rats that had consumed excessive amounts of sucrose during binge sessions (24h sucrose group). RIM had no effect in the $12 \mathrm{~h}$ sucrose groups because these animals did not exhibit a CPP (i.e., there was nothing to block). The elimination of a sucrose CPP across all three drug conditions (vehicle, RIM 1, and RIM 3) in all 12h sucrose groups matches our earlier report (Smail-Crevier et al., 2018), suggesting that the lack of a CPP in these animals is due to prior binge-like intake. We proposed, previously, that this behavior modifies brain reward systems, impacting the later expression of a CPP. Our current findings add to this discussion by indicating that excessive sucrose intake, itself, may also disrupt ECS function. That is, 28 days of sucrose consumption may have compromised ECS-linked reward processing, rendering animals susceptible to CB1R blockade in the CPP paradigm. This was manifested by the absence of a CPP in the $24 \mathrm{~h}$ sucrose, $3 \mathrm{mg} / \mathrm{kg}$ RIM group.

An alternative interpretation is that the lack of CPP in RIM-treated animals reflects alterations in neural mechanisms of anxiety in that sucrose bingeing increases anxiety-like behavior in rats (Avena, Bocarsly, et al., 2008), and the ECS plays an important role in regulating stress-related responses, such as anxiety (Morena et al., 2016). In support of this idea, the 24h sucrose group exhibited ECS changes in the MPFC and HPC, brain regions that are particularly vulnerable to stress manipulations. Finally, the fact that CB1R antagonism reduced sucrose consumption during conditioning, but did not block a CPP during testing (i.e., in rats who had no prior experience with sucrose), is consistent with evidence showing a dissociation in 
mechanisms that control these two processes (White \& Carr, 1985; Papp et al., 1991; Ågmo et al., 1995; Smail-Crevier et al., 2018).

To the extent that preclinical research is applicable to drug development, our findings suggest that cannabinoid compounds may be appropriate tools to treat BED. CB1Rs could be the most promising target in that $\mathrm{CB} 1 \mathrm{R}$ expression is elevated in eating disorder patients (Frieling et al., 2009). Moreover, CB1R antagonists reduce binge eating in both rats (Parylak et al., 2012; Scherma et al., 2013; Dore et al., 2014) and humans (Pataky et al., 2013), although at least one drug, SR141716 (Rimonabant), produces adverse events in BED patients (Pataky et al., 2013). Selective targets of peripheral, rather than central, CB1Rs may mitigate these negative side effects. As an example, AM6545, a peripherally-restricted CB1R antagonist, diminishes intake of a high fat-high sugar diet in mice, with no alteration in consumption of standard chow (Argueta \& DiPatrizio, 2017). In addition, cannabidiol, a CB1R antagonist with CB2R agonist activity, reduces sucrose intake in mice, an effect that is potentiated by CB1R blockade and inhibited by CB2R blockade (Bi et al., 2020). Currently, there are only two drugs approved by the US food and drug administration to treat BED: lisdexamfetamine (Vyvanse), an inactive prodrug promoting the release of monoamines (McElroy et al., 2015; Gasior et al., 2017), and dasotraline, a DA and norepinephrine reuptake inhibitor (Grilo et al., 2020). Lisdexamfetamine produces multiple adverse effects (Ward \& Citrome, 2018) and dasotraline was recently withdrawn from drug development. Given the paucity of alternative therapies for BED (Hutson et al., 2018), a strategic focus on ECS mechanisms may provide the best options for treating this disorder.

Finally, our study adds to the growing discussion concerning addictive-like properties of palatable food. On a general level, our model of sucrose bingeing shares a number of 
commonalities with drug addiction (Bocarsly \& Avena, 2013), including escalation of intake (Colantuoni et al., 2001), signs of somatic withdrawal (Colantuoni et al., 2002; Avena, Bocarsly, et al., 2008), and neurochemical changes in the NAc, such as increased DA release (Avena et al., 2005; Rada et al., 2005) and reduced DA D2 receptor availability (Colantuoni et al., 2001). Most telling, sucrose bingeing induces compulsive responding and electrophysiological changes in the bed nucleus of the stria terminalis (Maracle et al., 2019) that match behavioral and biological consequences of cocaine self-administration in rats (Krawczyk, Georges, et al., 2011; Krawczyk, Sharma, et al., 2011). Further, food craving in humans is associated with enhanced activity in the HPC, NAc, insula, and orbitofrontal cortex (Pelchat et al., 2004; Rolls \& McCabe, 2007), the same regions that are activated in drug craving (Koob \& Volkow, 2010; Everitt \& Robbins, 2016). In addition, future experiments will investigate possible sex differences in the effects of binge intake on ECS processes. Given that baseline levels of endocannabinoids and reactivity towards cannabinoids are sex-dependent (for review see Fattore \& Fratta, 2010; Craft et al., 2013), we may expect distinct patterns of ECS changes in males and females following excessive intake of palatable food. These differences may contribute, at least partially, to the higher prevalence of eating disorders in females than males (Bulik et al., 2015).

The ECS may provide a link between responses to palatable food and addictive behaviors, particularly as cannabinoid mechanisms are intimately linked to drug addiction (Silveira et al., 2017). In support of this idea, CB1R antagonism reduces the proportion of animals that transition from controlled to compulsive feeding in a mouse model of addictive-like eating (Mancino et al., 2015). At the same time, the concept of food addiction is not universally accepted (Ziauddeen et al., 2012; Westwater et al., 2016; Treasure et al., 2018), with a growing awareness that research should focus on identifying biological underpinnings of specific 
behavioral patterns that lead to excessive intake of food or drugs (Dileone et al., 2012). Our findings inform this line of investigation by highlighting how endocannabinoid mechanisms in brain reward regions may contribute to binge eating.

\section{Acknowledgments}

This project was supported by the Université de Strasbourg, Centre National de la Recherche Scientifique (CNRS), Programme International de Cooperation Scientifique (PICS CNRS), the French National Research Agency (ANR) through the Programme d'Investissement d'Avenir (ANR17-EURE-0022, Euridol, graduate school of pain) and the Natural Sciences and Engineering Research Council of Canada (NSERC). RB received a postdoctoral fellowship from the Fondation pour la Recherche Médicale (FRM). DN and GA received a PhD fellowship from the French Ministère de l'Enseignement Supérieur et de la Recherche and DN received a fourth year $\mathrm{PhD}$ fellowship from the Fonds Paul Mendel. We thank Lihua Xue for help with behavioral testing and Alicia Jung for help with biochemical analysis.

\section{Author's contribution}

Conceptualization: DN, MCO, KB designed the experiments and wrote the paper. Investigation: DN, VA, DF, KB performed the experiments; DN, RB, KB analyzed the data and designed the figures; GA and MCO conducted statistical analysis; YG, VA contributed to the design and analysis of the mass spectrometry experiment. Supervision and funding acquisition: MCO and KB supervised the study and obtained the funding. All authors approved the final version of the manuscript.

Conflict of interest. The authors declare no conflict of interest. 


\section{Data availability statement}

All the data supporting the findings of this study can be provided upon request from the corresponding author. 


\section{References}

Abel, E.L. (1975) Cannabis: effects on hunger and thirst. Behav. Biol., 15, 255-281.

Achterberg, E. J. M., van Swieten, M. M. H., Driel, N. V., Trezza, V., \& Vanderschuren, L. (2016). Dissociating the role of endocannabinoids in the pleasurable and motivational properties of social play behaviour in rats. Pharmacol Res, 110, 151-158.

Ågmo, A., Galvan, A., \& Talamantes, B. (1995) Reward and reinforcement produced by drinking sucrose: Two processes that may depend on different neurotransmitters. Pharmacol. Biochem. Behav., 52, 403-414.

Argueta, D.A. \& DiPatrizio, N. V (2017) Peripheral endocannabinoid signaling controls hyperphagia in western diet-induced obesity. Physiol. Behav., 171, 32-39.

Arnone, M., Maruani, J., Chaperon, F., Thiébot, M.H., Poncelet, M., Soubrié, P., \& Le Fur, G. (1997) Selective inhibition of sucrose and ethanol intake by SR 141716, an antagonist of central cannabinoid (CB1) receptors. Psychopharmacology (Berl)., 132, 104-106.

Association, A.P. (2013) DSM 5, American Journal of Psychiatry.

Avena, N.M. (2007) Examining the Addictive-Like Properties of Binge Eating Using an Animal Model of Sugar Dependence. Exp. Clin. Psychopharmacol., 15, 481-491.

Avena, N.M. (2010) The study of food addiction using animal models of binge eating. Appetite, 55, 734-737.

Avena, N.M., Bocarsly, M.E., Rada, P., Kim, A., \& Hoebel, B.G. (2008) After daily bingeing on a sucrose solution, food deprivation induces anxiety and accumbens dopamine/acetylcholine imbalance. Physiol. Behav., 94, 309-315.

Avena, N.M., Long, K.A., \& Hoebel, B.G. (2005) Sugar-dependent rats show enhanced responding for sugar after abstinence: Evidence of a sugar deprivation effect. Physiol. Behav., 84, 359-362.

Avena, N.M., Rada, P., \& Hoebel, B.G. (2008) Evidence for sugar addiction: Behavioral and neurochemical effects of intermittent, excessive sugar intake. Neurosci. Biobehav. Rev., 32, 20-39.

Bello, N.T., Coughlin, J.W., Redgrave, G.W., Ladenheim, E.E., Moran, T.H., \& Guarda, A.S. (2012) Dietary conditions and highly palatable food access alter rat cannabinoid receptor expression and binding density. Physiol. Behav., 105, 720-726.

Bi, G.H., Galaj, E., He, Y., \& Xi, Z.X. (2020) Cannabidiol inhibits sucrose self-administration 
by CB1 and CB2 receptor mechanisms in rodents. Addict. Biol., 25, e12783.

Blanco-Gandía, M.C., Aracil-Fernández, A., Montagud-Romero, S., Aguilar, M.A., Manzanares, J., Miñarro, J., \& Rodríguez-Arias, M. (2017) Changes in gene expression and sensitivity of cocaine reward produced by a continuous fat diet. Psychopharmacology (Berl)., 234, 23372352.

Blanco-Gandía, M.C., Cantacorps, L., Aracil-Fernández, A., Montagud-Romero, S., Aguilar, M.A., Manzanares, J., Valverde, O., Miñarro, J., \& Rodríguez-Arias, M. (2017) Effects of bingeing on fat during adolescence on the reinforcing effects of cocaine in adult male mice. Neuropharmacology, 113, 31-44.

Blasio, A., Rice, K. C., Sabino, V., \& Cottone, P. (2014). Characterization of a shortened model of diet alternation in female rats: effects of the CB1 receptor antagonist rimonabant on food intake and anxiety-like behavior. Behav Pharmacol, 25, 609-617.

Bocarsly, M.E. \& Avena, N.M. (2013) Animal Models of Binge Eating Palatable Foods: Emergence of Addiction-Like Behaviors and Brain Changes in the Rat. pp. 179-191.

Bulik, C., Yilmaz, Z., \& HArdaway, A. (2015) Genetics and epigenetics of eating disorders. Adv. Genomics Genet., 5, 131-150.

Chaperon, F., Soubrié, P., Puech, A. J., \& Thiébot, M.-H. (1998). Involvement of central cannabinoid (CB 1 ) receptors in the establishment of place conditioning in rats. Psychopharmacology, 135, 324-332.

Ceccarini, J., Weltens, N., Ly, H.G., Tack, J., Van Oudenhove, L., \& Van Laere, K. (2016) Association between cerebral cannabinoid 1 receptor availability and body mass index in patients with food intake disorders and healthy subjects: a [(18)F]MK-9470 PET study. Transl. Psychiatry, 6, e853.

Colantuoni, C., Rada, P., McCarthy, J., Patten, C., Avena, N.M., Chadeayne, A., \& Hoebel, B.G. (2002) Evidence That Intermittent, Excessive Sugar Intake Causes Endogenous Opioid Dependence. Obes. Res., 10, 478-488.

Colantuoni, C., Schwenker, J., McCarthy, J., Rada, P., Ladenheim, B., Cadet, J.-L., Schwartz, G.J., Moran, T.H., \& Hoebel, B.G. (2001) Excessive sugar intake alters binding to dopamine and mu-opioid receptors in the brain. Neuroreport, 12, 3549-3552.

Colombo, G., Serra, S., Brunetti, G., Gomez, R., Melis, S., Vacca, G., Carai, M.A., \& Gessa, G. (2002) Stimulation of voluntary ethanol intake by cannabinoid receptor agonists in ethanol- 
preferring sP rats. Psychopharmacology (Berl)., 159, 181-187.

Craft, R.M., Marusich, J.A., \& Wiley, J.L. (2013) Sex differences in cannabinoid pharmacology: A reflection of differences in the endocannabinoid system? Life Sci., 92, 476-481.

Dazzi, L., Talani, G., Biggio, F., Utzeri, C., Lallai, V., Licheri, V., Lutzu, S., Mostallino, M.C., Secci, P.P., Biggio, G., \& Sanna, E. (2014) Involvement of the cannabinoid CB1 receptor in modulation of dopamine output in the prefrontal cortex associated with food restriction in rats. PLoS One, 9, e92224.

De Carvalho, C. R., Pamplona, F. A., Cruz, J. S., \& Takahashi, R. N. (2014). Endocannabinoids underlie reconsolidation of hedonic memories in Wistar rats. Psychopharmacology (Berl), 231, 1417-1425.

De Vries, T. J., Shaham, Y., Homberg, J. R., Crombag, H., Schuurman, K., Dieben, J., Vanderschuren, L. J., \& Schoffelmeer, A. N. (2001). A cannabinoid mechanism in relapse to cocaine seeking. Nat Med, 7, 1151-1154.

Dileone, R.J., Taylor, J.R., \& Picciotto, M.R. (2012) iThe drive to eat: Comparisons and distinctions between mechanisms of food reward and drug addiction. Nat. Neurosci., 15, 1330-1335.

Dore, R., Valenza, M., Wang, X., Rice, K.C., Sabino, V., \& Cottone, P. (2014) The inverse agonist of CB1 receptor SR141716 blocks compulsive eating of palatable food. Addict. Biol., 19, 849-861.

Everitt, B.J. \& Robbins, T.W. (2016) Drug Addiction: Updating Actions to Habits to Compulsions Ten Years On. Annu. Rev. Psychol., 67, 23-50.

Fattore, L. \& Fratta, W. (2010) How important are sex differences in cannabinoid action? Br. J. Pharmacol., 160, 544-548.

Frieling, H., Albrecht, H., Jedtberg, S., Gozner, A., Lenz, B., Wilhelm, J., Hillemacher, T., de Zwaan, M., Kornhuber, J., \& Bleich, S. (2009) Elevated cannabinoid 1 receptor mRNA is linked to eating disorder related behavior and attitudes in females with eating disorders. Psychoneuroendocrinology, 34, 620-624.

Gaetani, S., Kaye, W.H., Cuomo, V., \& Piomelli, D. (2008) Role of endocannabinoids and their analogues in obesity and eating disorders. Eat. Weight Disord., 13, e42-8.

Gasior, M., Hudson, J., Quintero, J., Ferreira-Cornwell, M.C., Radewonuk, J., \& McElroy, S.L. (2017) A Phase 3, Multicenter, Open-Label, 12-Month Extension Safety and Tolerability 
Binge sucrose regulation of the ECS

Trial of Lisdexamfetamine Dimesylate in Adults With Binge Eating Disorder. J. Clin.

Psychopharmacol., 37, 315-322.

Grenier, P., Mailhiot, M.C., Cahill, C.M., \& Olmstead, M.C. (2019) Blockade of dopamine D1 receptors in male rats disrupts morphine reward in pain naïve but not in chronic pain states. J. Neurosci. Res., $00,1-12$.

Grilo, C.M., McElroy, S.L., Hudson, J.I., Tsai, J., Navia, B., Goldman, R., Deng, L., Kent, J., \& Loebel, A. (2020) Efficacy and safety of dasotraline in adults with binge-eating disorder: A randomized, placebo-controlled, fixed-dose clinical trial. CNS Spectr., 19, 1-10.

Hernandez, G. \& Cheer, J.F. (2012) Effect of CB1 receptor blockade on food-reinforced responding and associated nucleus accumbens neuronal activity in rats. J. Neurosci., 32, $11467-11477$.

Hudson, J.I., Hiripi, E., Pope, H.G., \& Kessler, R.C. (2007) The Prevalence and Correlates of Eating Disorders in the National Comorbidity Survey Replication. Biol. Psychiatry, 61, $348-358$.

Hutson, P.H., Balodis, I.M., \& Potenza, M.N. (2018) Binge-eating disorder: Clinical and therapeutic advances. Pharmacol. Ther., 182, 15-27.

Jarbe, T. U., Gifford, R. S., \& Makriyannis, A. (2010). Antagonism of (9)-THC induced behavioral effects by rimonabant: time course studies in rats. Eur J Pharmacol, 648, 133138.

Joseph, M.H. \& Hodges, H. (1990) Lever pressing for food reward and changes in dopamine turnover and uric acid in rat caudate and nucleus accumbens studied chronically by in vivo voltammetry. J. Neurosci. Methods, 34, 143-149.

Kelley, A.E., Baldo, B.A., Pratt, W.E., \& Will, M.J. (2005) Corticostriatal-hypothalamic circuitry and food motivation: Integration of energy, action and reward. Physiol. Behav., 86, 773-795.

Kessler, R.C., Berglund, P.A., Chiu, W.T., Deitz, A.C., Hudson, J.I., Shahly, V., AguilarGaxiola, S., Alonso, J., Angermeyer, M.C., Benjet, C., Bruffaerts, R., de Girolamo, G., de Graaf, R., Maria Haro, J., Kovess-Masfety, V., O’Neill, S., Posada-Villa, J., Sasu, C., Scott, K., Viana, M.C., \& Xavier, M. (2013) The prevalence and correlates of binge eating disorder in the World Health Organization World Mental Health Surveys. Biol. Psychiatry, 73, 904-914. 
Khaleghzadeh-Ahangar, H. \& Haghparast, A. (2015) Intra-accumbal CB1 receptor blockade reduced extinction and reinstatement of morphine. Physiol. Behav., 149, 212-219.

Kirkham, T.C., Williams, C.M., Fezza, F., \& Di Marzo, V. (2002) Endocannabinoid levels in rat limbic forebrain and hypothalamus in relation to fasting, feeding and satiation: Stimulation of eating by 2-arachidonoyl glycerol. Br. J. Pharmacol., 136, 550-557.

Koch, M., Varela, L., Kim, J.G., Kim, J.D., Hernández-Nuño, F., Simonds, S.E., Castorena, C.M., Vianna, C.R., Elmquist, J.K., Morozov, Y.M., Rakic, P., Bechmann, I., Cowley, M.A., Szigeti-Buck, K., Dietrich, M.O., Gao, X.-B., Diano, S., \& Horvath, T.L. (2015) Hypothalamic POMC neurons promote cannabinoid-induced feeding. Nature, 519, 45-50.

Koob, G.F. \& Volkow, N.D. (2010) Neurocircuitry of addiction. Neuropsychopharmacology, 35, $217-238$.

Krawczyk, M., Georges, F., Sharma, R., Mason, X., Berthet, A., Bézard, E., \& Dumont, É.C. (2011) Double-dissociation of the catecholaminergic modulation of synaptic transmission in the oval bed nucleus of the stria terminalis. J. Neurophysiol., 105, 145-153.

Krawczyk, M., Sharma, R., Mason, X., de Backer, J., Jones, A.A., \& Dumont, É.C. (2011) A switch in the neuromodulatory effects of dopamine in the oval bed nucleus of the stria terminalis associated with cocaine self-administration in rats. J. Neurosci., 31, 8928-8935.

Li, B., Matter, E.K., Hoppert, H.T., Grayson, B.E., Seeley, R.J., \& Sandoval, D.A. (2014) Identification of optimal reference genes for RT-qPCR in the rat hypothalamus and intestine for the study of obesity. Int. J. Obes., 38, 192-197.

Maldonado, R., Valverde, O., \& Berrendero, F. (2006) Involvement of the endocannabinoid system in drug addiction. Trends Neurosci., 29, 225-232.

Mancino, S., Burokas, A., Gutiérrez-Cuesta, J., Gutiérrez-Martos, M., Martín-García, E., Pucci, M., Falconi, A., D’Addario, C., Maccarrone, M., \& Maldonado, R. (2015) Epigenetic and proteomic expression changes promoted by eating addictive-like behavior. Neuropsychopharmacology, 40, 2788-2800.

Manduca, A., Lassalle, O., Sepers, M., Campolongo, P., Cuomo, V., Marsicano, G., Kieffer, B., Vanderschuren, L.J.M.J., Trezza, V., \& Manzoni, O.J.J. (2016) Interacting Cannabinoid and Opioid Receptors in the Nucleus Accumbens Core Control Adolescent Social Play. Front. Behav. Neurosci., 10, 211.

Maracle, A.C., Normandeau, C.P., Dumont, É.C., \& Olmstead, M.C. (2019) Dopamine in the 
oval bed nucleus of the stria terminalis contributes to compulsive responding for sucrose in rats. Neuropsychopharmacology, 44, 381-389.

McElroy, S.L., Hudson, J.I., Mitchell, J.E., Wilfley, D., Ferreira-Cornwell, M.C., Gao, J., Wang, J., Whitaker, T., Jonas, J., \& Gasior, M. (2015) Efficacy and Safety of Lisdexamfetamine for Treatment of Adults With Moderate to Severe Binge-Eating Disorder. JAMA Psychiatry, 72, 235-246.

Monteleone, P., Matias, I., Martiadis, V., De Petrocellis, L., Maj, M., \& Di Marzo, V. (2005) Blood levels of the endocannabinoid anandamide are increased in anorexia nervosa and in binge-eating disorder, but not in bulimia nervosa. Neuropsychopharmacology, 30, 12161221.

Morena, M., Patel, S., Bains, J.S., \& Hill, M.N. (2016) Neurobiological Interactions Between Stress and the Endocannabinoid System. Neuropsychopharmacology, 41, 80-102.

Papp, M., Willner, P., \& Muscat, R. (1991) An animal model of anhedonia: attenuation of sucrose consumption and place preference conditioning by chronic unpredictable mild stress. Psychopharmacology (Berl)., 104, 255-259.

Parylak, S.L., Cottone, P., Sabino, V., Rice, K.C., \& Zorrilla, E.P. (2012) Effects of CB1 and CRF1 receptor antagonists on binge-like eating in rats with limited access to a sweet fat diet: lack of withdrawal-like responses. Physiol. Behav., 107, 231-242.

Pataky, Z., Gasteyger, C., Ziegler, O., Rissanen, A., Hanotin, C., \& Golay, A. (2013) Efficacy of rimonabant in obese patients with binge eating disorder. Exp. Clin. Endocrinol. Diabetes, 121, 20-26.

Paxinos, G. \& Watson, C. (2007) The Rat Brain in Stereotaxic Coordinates. Elsevier. Peciña, S. \& Berridge, K.C. (2005) Hedonic hot spot in nucleus accumbens shell: Where do $\mu$ Opioids cause increased hedonic impact of sweetness? J. Neurosci., 25, 11777-11786.

Pelchat, M.L., Johnson, A., Chan, R., Valdez, J., \& Ragland, J.D. (2004) Images of desire: foodcraving activation during fMRI. Neuroimage, 23, 1486-1493.

Phillips, A.G., Atkinson, L.J., Blackburn, J.R., \& Blaha, C.D. (1993) Increased extracellular dopamine in the nucleus accumbens of the rat elicited by a conditional stimulus for food: An electrochemical study. Can. J. Physiol. Pharmacol., 71, 387-393.

Rada, P., Avena, N.M., \& Hoebel, B.G. (2005) Daily bingeing on sugar repeatedly releases dopamine in the accumbens shell. Neuroscience, 134, 737-744. 
Rios, C., Gomes, I., \& Devi, L.A. (2006) mu opioid and CB1 cannabinoid receptor interactions: reciprocal inhibition of receptor signaling and neuritogenesis. Br. J. Pharmacol., 148, 387395.

Rodriguez, J.J., Mackie, K., \& Pickel, V.M. (2001) Ultrastructural localization of the CB1 cannabinoid receptor in mu-opioid receptor patches of the rat Caudate putamen nucleus. $J$. Neurosci., 21, 823-833.

Rolls, E.T. \& McCabe, C. (2007) Enhanced affective brain representations of chocolate in cravers vs. non-cravers. Eur. J. Neurosci., 26, 1067-1076.

Satta, V., Scherma, M., Piscitelli, F., Usai, P., Castelli, M.P., Bisogno, T., Fratta, W., \& Fadda, P. (2018) Limited Access to a High Fat Diet Alters Endocannabinoid Tone in Female Rats. Front. Neurosci., 12, 40.

Scherma, M., Fattore, L., Satta, V., Businco, F., Pigliacampo, B., Goldberg, S.R., Dessi, C., Fratta, W., \& Fadda, P. (2013) Pharmacological modulation of the endocannabinoid signalling alters binge-type eating behaviour in female rats. Br. J. Pharmacol., 169, 820833.

Schmid, P.C., Krebsbach, R.J., Perry, S.R., Dettmer, T.M., Maasson, J.L., \& Schmid, H.H.O. (1995) Occurrence and postmortem generation of anandamide and other long-chain $N$ acylethanolamines in mammalian brain. FEBS Lett., 375, 117-120.

Silberberg, G., Baruch, K., \& Navon, R. (2009) Detection of stable reference genes for real-time PCR analysis in schizophrenia and bipolar disorder. Anal. Biochem., 391, 91-97.

Silveira, M.M., Arnold, J.C., Laviolette, S.R., Hillard, C.J., Celorrio, M., Aymerich, M.S., \& Adams, W.K. (2017) Seeing through the smoke: Human and animal studies of cannabis use and endocannabinoid signalling in corticolimbic networks. Neurosci. Biobehav. Rev., 76, $380-395$.

Simiand, J., Keane, M., Keane, P.E., \& Soubrié, P. (1998) SR 141716, a CB1 cannabinoid receptor antagonist, selectively reduces sweet food intake in marmoset. Behav. Pharmacol., 9, 179-181.

Smail-Crevier, R.L., Maracle, A.C., Wash, S.I.J., \& Olmstead, M.C. (2018) Binge-like intake of sucrose reduces the rewarding value of sucrose in adult rats. Physiol. Behav., 194, 420-429.

Solinas, M., Justinova, Z., Goldberg, S.R., \& Tanda, G. (2006) Anandamide administration alone and after inhibition of fatty acid amide hydrolase (FAAH) increases dopamine levels in the 
nucleus accumbens shell in rats. J. Neurochem., 98, 408-419.

Solmi, F., Hotopf, M., Hatch, S.L., Treasure, J., \& Micali, N. (2016) Eating disorders in a multiethnic inner-city UK sample: prevalence, comorbidity and service use. Soc. Psychiatry Psychiatr. Epidemiol., 51, 369-381.

Sperlágh, B., Windisch, K., Andó, R.D., \& Sylvester Vizi, E. (2009) Neurochemical evidence that stimulation of $\mathrm{CB} 1$ cannabinoid receptors on GABAergic nerve terminals activates the dopaminergic reward system by increasing dopamine release in the rat nucleus accumbens. Neurochem. Int., 54, 452-457.

Stojek, M.M.K. \& MacKillop, J. (2017) Relative reinforcing value of food and delayed reward discounting in obesity and disordered eating: A systematic review. Clin. Psychol. Rev., 55, 1-11.

Thoeni, S., Loureiro, M., O’Connor, E.C., \& Lüscher, C. (2020) Depression of Accumbal to Lateral Hypothalamic Synapses Gates Overeating. Neuron, 107, 158-172.

Treasure, J., Leslie, M., Chami, R., \& Fernández-Aranda, F. (2018) Are trans diagnostic models of eating disorders fit for purpose? A consideration of the evidence for food addiction. Eur. Eat. Disord. Rev., 26, 83-91.

Trezza, V., Damsteegt, R., Manduca, A., Petrosino, S., Van Kerkhof, L.W.M., Pasterkamp, R.J., Zhou, Y., Campolongo, P., Cuomo, V., Di Marzo, V., \& Vanderschuren, L.J.M.J. (2012) Endocannabinoids in amygdala and nucleus accumbens mediate social play reward in adolescent rats. J. Neurosci., 32, 14899-14908.

Trezza, V. \& Vanderschuren, L.J.M.J. (2008) Bidirectional cannabinoid modulation of social behavior in adolescent rats. Psychopharmacology (Berl)., 197, 217-227.

Ward, K. \& Citrome, L. (2018) Lisdexamfetamine: chemistry, pharmacodynamics, pharmacokinetics, and clinical efficacy, safety, and tolerability in the treatment of binge eating disorder. Expert Opin. Drug Metab. Toxicol., 14, 229-238.

Wei, D., Lee, D.Y., Cox, C.D., Karsten, C.A., Penagarikano, O., Geschwind, D.H., Gall, C.M., \& Piomelli, D. (2015) Endocannabinoid signaling mediates oxytocin-driven social reward. Proc. Natl. Acad. Sci. U. S. A., 112, 14084-14089.

Westwater, M.L., Fletcher, P.C., \& Ziauddeen, H. (2016) Sugar addiction: the state of the science. Eur. J. Nutr., 55, 55-69.

White, N.M. \& Carr, G.D. (1985) The conditioned place preference is affected by two 
independent reinforcement processes. Pharmacol. Biochem. Behav., 23, 37-42.

Williams, C.M., Rogers, P.J., \& Kirkham, T.C. (1998) Hyperphagia in pre-fed rats following oral 89-THC. Physiol. Behav., 65, 343-346.

Wong, K.J., Wojnicki, F.H.W., \& Corwin, R.L.W. (2009) Baclofen, raclopride, and naltrexone differentially affect intake of fat/sucrose mixtures under limited access conditions.

Pharmacol. Biochem. Behav., 92, 528-536.

Yu, L.-L., Zhou, S.-J., Wang, X.-Y., Liu, J.-F., Xue, Y.-X., Jiang, W., \& Lu, L. (2011). Effects of cannabinoid $\mathrm{CB}_{1}$ receptor antagonist rimonabant on acquisition and reinstatement of psychostimulant reward memory in mice. Behavioural brain research, 217, 111-116.

Ziauddeen, H., Farooqi, I.S., \& Fletcher, P.C. (2012) Obesity and the brain: How convincing is the addiction model? Nat. Rev. Neurosci., 13, 279-286. 


\section{Figure Captions}

Figure 1. Timeline of experimental procedures. Four separate experiments examined: ECS alterations following sucrose bingeing (Experiment 1), the effect of RIM on sucrose bingeing on days 1-5 and 24-28 of a 28-day sucrose access protocol (Experiment 2), the effect of RIM on a sucrose CPP (Experiment 3), and the effect of RIM on a sucrose CPP following sucrose bingeing (Experiment 4). CPP: conditioned place preference; ECS: endocannabinoid system; RIM: rimonabant.

Figure 2. Intermittent access to sucrose (12h sucrose) induces binge-like intake. (A) Solution intake (mL) per body weight ( $\mathrm{g}$ ) during the first hour of access across 28 days. (B) Daily solution intake (mL) per body weight (g) across sessions. (C) Daily food intake (g) per body weight (g) across sessions. (D) Body weight (g) across sessions. Data are presented as group means $( \pm$ SEM).

Figure 3. Intermittent access to sucrose modulates endocannabinoid gene expression in the NAc. Gene expression was examined in the PFC (n=7-8/group), NAc (5-10/group), DS (n=68/group), and HPC (n=7-10/group). (A) CB1R gene expression was significantly increased in the NAc of the $12 \mathrm{~h}$ sucrose group compared to the other groups. CB2 gene expression remained unchanged. (B) FAAH gene expression was significantly reduced in the NAc of the $24 \mathrm{~h}$ sucrose group. (C) DAGLa gene expression was significantly decreased in the NAc of both $12 \mathrm{~h}$ and $24 \mathrm{~h}$ sucrose groups. Data are presented as group means (+SEM). * $p<.05, * * p<.01, * * * p<.001$ compared to $12 \mathrm{~h}$ food; \# $p<.05$, compared to $24 \mathrm{~h}$ sucrose group. CB1/2: cannabinoid receptor 1/2; DS: dorsal striatum. HPC: hippocampus; NAc: nucleus accumbens; PFC: prefrontal cortex. Figure 4. Intermittent access to sucrose alters endocannabinoid levels. Endocannabinoid levels were examined in the PFC, NAc, DS, and HPC. (A) AEA levels were significantly 
increased in the PFC of the 12h sucrose group. (B) 2-AG levels were significantly lower in the HPC of the $12 \mathrm{~h}$ sucrose group. Data are presented as group means $(+\mathrm{SEM}) .{ }^{*} p<.01$ : compare to 12h food. 2AG: 2-arachidonoylglycerol; AEA: anandamide; DS: dorsal striatum; HPC: hippocampus; NAc: nucleus accumbens; PFC: prefrontal cortex.

Figure 5. Rimonabant decreases binge-like intake of sucrose. (A) Solution intake (mL) per body weight (g) during the first hour of access across 28 days (left), and total intake on the drug injection days (days 1-5 and 24-28) for the 12h sucrose group (right). (B) Daily solution intake (mL) per body weight (g) (top), daily food intake (g) per body weight (g) (middle), body weight (g) (bottom) across sessions, for animals under a $12 \mathrm{~h}$ (right panels) or 24h sucrose access (left panels) protocol. Data are presented as group means ( $(\mathrm{SEM})$. RIM 1: rimonabant $1 \mathrm{mg} / \mathrm{kg}$; RIM 3: rimonabant $3 \mathrm{mg} / \mathrm{kg}$; Veh: vehicle.

Figure 6. Rimonabant blocks a sucrose CPP following excessive sucrose consumption. (A) Effect of RIM on total solution intake across conditioning sessions (Experiment 3). (B) Time spent in each compartment during CPP testing (Experiment 3). (C) Effect of RIM on total solution intake across conditioning sessions, following sucrose consumption sessions (Experiment4). (D) Time spent in each compartment during CPP testing following sucrose consumption sessions (Experiment 4). Data are presented as group means $(+\mathrm{SEM}) . * p<.05, * *$ $p<.01$. CPP: conditioned place preference; RIM 1: rimonabant $1 \mathrm{mg} / \mathrm{kg}$; RIM 3: rimonabant 3 $\mathrm{mg} / \mathrm{kg}$; Veh: vehicle

Table 1. Primer sequences for reference (Rplp0) and endocannabinoid system genes. 